\title{
Electric Stress in Power Electronics Applications
}

\author{
A. Carboni \\ $D E I B$ \\ Politecnico di Milano \\ Milan, Italy \\ alberto.carboni@polimi.it
}

\author{
K. ElShawarby, G. M. Foglia, \\ R. Perini, A. Di Gerlando \\ DENG \\ Politecnico di Milano \\ Milan, Italy
}

$\{$ khaled.elshawarby, gianmaria.foglia,

roberto.perini, antonino.digerlando\} @ polimi.it

\author{
E. Ragaini \\ Electrification Products \\ ABB S.p.A. \\ Bergamo, Italy \\ enrico.ragaini @it.abb.com
}

\begin{abstract}
Voltage quality is a major issue for several appliances in today's electric power systems. Distorted voltage waveforms caused by power electronics devices can dramatically reduce the useful life of dielectrics, e.g., filter capacitor banks or power factor correction capacitor banks.

This paper aims at investigating how different power electronics control schemes can affect the dielectric ageing and, in doing so, it aims at providing a feasible and sustainable electric stress monitoring framework. The two considered control schemes adopted are the classical SPWM and SPWM with sequential command.

Index Terms-Dielectric Ageing, Electric Stress, Converter Se-
\end{abstract} quential Command, Sinusoidal Pulse Width Modulation (SPWM)

\section{INTRODUCTION}

The increasing number of electronic equipment that can affect voltage quality along with a deregulated electricity market have made Power Quality (PQ) a field of interest both in the research and industrial field [1].

It is well known, in fact, that distorted waveforms generated by power electronics can dramatically reduce the reliability of insulation systems i.e. decrease the dielectric life expectancy. Two kinds of stress mainly affect dielectrics: thermal stress caused by current distortion and electric stresses caused by voltage distortion.

This paper focuses on electric stress as it proves to be more critical for dielectrics than thermal stress [2]-[6].

In particular, this paper aims at:

- comparing the effect on capacitor dielectric ageing of a well-established modulation technique, i.e., Sinusoidal Pulse Width Modulation (SPWM), versus sequential command SPWM [7]. Classical control strategies, in fact, may prove sub-optimal when using the electric stress as a benchmark.

- defining a feasible and sustainable electric stress monitoring framework. In fact, the main problem in dielectric ageing monitoring is the great variability of the life behaviour depending on the operating conditions [8], [9]. This would require, in fact, large-scale validation and testing campaigns for all the different possible installations, thus viable alternative solutions have to be looked for.

The results reported in this paper are part of the activities of the ABB \& Politecnico di Milano Joint Research Centre.
With this in mind, the digitalization of the electric grid has opened the way to new possible applications in PQ monitoring solutions, e.g., already installed electric equipment is becoming smart, thus able to communicate data to the cloud.

In particular, smart Circuit Breakers (CBs) [10], [11] are a possible solution for distributed monitoring networks with minimum impact on existing plant.

The rest of this work is organized as follows. Section II shows the dielectric ageing monitoring framework, lists the monitored coefficients and residual useful life model. Section III introduces the system to be studied, focuses on sequential command, a control scheme for parallel power converters. It derives the approximated expression of the DC bus voltage under SPWM modulation and shows the ripple compensation through the sequential phase shift of the carrier signals. Section IV compares SPWM and sequential command. Section V compares the dielectric stress induced by sequential command and SPWM in a wind energy system.

\section{Dielectric Ageing}

The dielectric ageing monitoring framework is implemented in the platform described in [10].

The framework architecture consists in a physical layer and the cloud layer.

a) The physical layer: In this layer, the smart CBs [10] collect data from the field and aggregate data in the relevant indices to be monitored, as explained below. Field processing has the advantage of limiting the amount of data to be sent and stored on the cloud.

In particular, only the indices related to the electric stress, i.e., voltage distortion, are considered as it proves to be much more critical for dielectric as opposed to thermal stress, i.e., current distortion [2].

b) The cloud layer: In this layer, the aggregated data sent by the CBs are stored and used together to monitor the residual useful life of the monitored dielectric. At the moment, given the few data collected the cloud layer is still being populated and, thus, not further discussed.

The monitored coefficients are derived from the one proposed in [3] and modified to fit the DC waveform of the capacitor voltage. They are: 
- Peak coefficient. It is the ratio between the maximum value of the voltage, $\max (v(t))$, and its rated value $V_{d_{n}}$.

$$
K_{p}=\frac{\max (v(t))}{V_{d_{n}}}
$$

- Average coefficient. It is defined in two different ways depending on the installation supply. In DC it is the ratio between the computed mean value and the theoretical one $V_{d_{n}}$, while in AC it considers rms values instead of mean values.

$$
K_{m}=\frac{\operatorname{mean}(v(t))}{V_{d_{n}}}
$$

- Shape coefficient. It is the ratio between the harmonics amplitude $V_{h}$ and the DC one $V_{d_{n}}$ weighted by the harmonic order $h$. This coefficient takes into account the presence of steep slopes, i.e., high derivatives, in the voltage waveform

$$
K_{s}=\sqrt{\sum_{h=1}^{N} h^{2} \frac{V_{h}^{2}}{V_{d_{n}}^{2}}}
$$

All the coefficients (1), (2), (3) are monitored over time and stored. The final aim, in fact, is to have a robust database to describe the ageing curves of dielectrics operating in similar conditions.

The simplest model, in fact, that can be used to describe the expected insulation lifetime $L$ is, according to [3]:

$$
L=L_{0} K_{p}^{-n_{p}} K_{m}^{-n_{m}} K_{s}^{-n_{s}}
$$

where $L_{0}, n_{P}, n_{m}$ and $n_{s}$ are adjustable parameters.

Because of the aforementioned adjustable parameters, up to now, the definition of robust ageing curves is a main issue because of the high variability of the coefficient characterizing the resulting curves [4], [8], [9]. Therefore, testing campaigns proved to be unfit for purpose, they simply required a too large training set to be statistically-speaking robust. From this perspective, this work proposes a sustainable solution for the future.

\section{Mathematical Model and Case Study}

\section{A. System Description}

In order to consider voltage stresses due to the different modulation techniques and the effect on the ageing coefficients, the plant shown in Fig. 1 is considered. It consists of a three-module Axial Flux Permanent Magnet Synchronous Machine (AFPMSM) which is directly driven (DD) by the wind turbine (WT). Each module is connected to a $1.6 \mathrm{kV}$ DC bus via a dedicated SPWM driven two-level IGBT rectifier (PC1, PC2, PC3). All the two-level converters are paralleled at the DC side.

Table I reports the rated data of one module of the machine. The machine is isotropic, thus the inductances of $d$ and $q$ axes are equal.
TABLE I

SINGLE MODULE AXIAL FLUX PERMANENT MAGNET MACHINE NAMEPLATE DATA

\begin{tabular}{|l|c|}
\hline$P_{n}[\mathrm{MW}], I_{n}[\mathrm{~A}]$ & $1.0,713$ \\
\hline Rated frequency $f_{\text {rated }}[\mathrm{Hz}]$ & 14.73 \\
\hline Rated Speed $\Omega_{n}[\mathrm{rpm}]$ & 17 \\
\hline $\begin{array}{l}\text { Line to neutral EMF at rated speed }[\mathrm{V}] \\
\text { (sinusoidal waveform) }\end{array}$ & 435 \\
\hline Phase resistance $R[\mathrm{~m} \Omega]$ & 14.59 \\
\hline Synchronous inductance $(d, q$ axes) $L[\mathrm{mH}]$ & 4.321 \\
\hline Rotor ext. diameter $[\mathrm{m}]$, Axial length $[\mathrm{m}]$ & $5.00,0.603$ \\
\hline DC bus Capacitance $[\mathrm{mF}]$, rated voltage $\left[V_{d n}\right]$ & 10,1600 \\
\hline No damping cage & \\
\hline
\end{tabular}

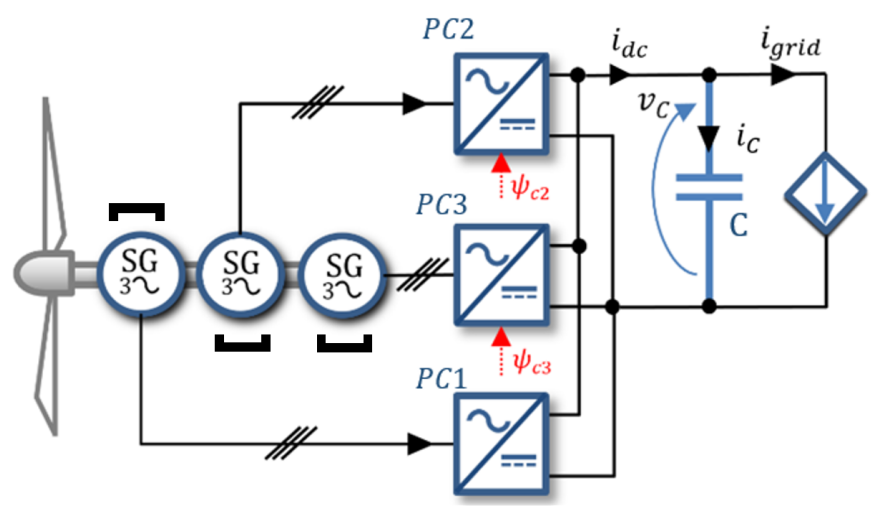

Fig. 1. A DD three-module AFPMSG machine: each module is connected to a two-level converter. All converters are connected in parallel on the DC side. The grid is modelled as current-controlled source $i_{\text {grid }}$.

Finally, the dielectric of interest is the DC bus capacitor $(C)$. The DC bus voltage $v_{C}$ is kept constant at its nominal value, $V_{d n}$, by the grid-side converter which is modelled as a current controlled source $i_{\text {grid }}$.

It is assumed the ripple injection is only due to the machineside converters, i.e., the grid converter does not inject any additional ripple to the DC bus.

\section{B. DC bus Voltage Expression under SPWM Modulation}

In [7] the approach of sequential command applied to SPWM modulation was proposed. The expression for the DC side current was developed. The idea of sequential command consists of shifting the carrier signals of neighbouring converters in order to eliminate some chosen harmonics and decrease the ripple content in $i_{d c}$.

Fig. 2 shows the proposed shift $\left(\psi_{c_{1}}-\psi_{c_{2}}-\psi_{c_{3}}\right)$ introduced in the triangular carrier signal for the three parallel converters. The shift $\psi_{c_{1}}$ is kept equal to zero while the carrier signals of the other two converters are shifted by $120^{\circ}, 240^{\circ}$ respectively.

To simplify the analysis, assume that all the ripple components of $i_{d c}$ flow in $C$.

$$
v_{C}=\frac{1}{C} \int i_{C} d t \simeq \frac{1}{C} \int i_{d c_{\text {ripple }}} d t
$$

Substituting the expression of the approximated DC side current, developed in [7], in (5), an approximate expression of 


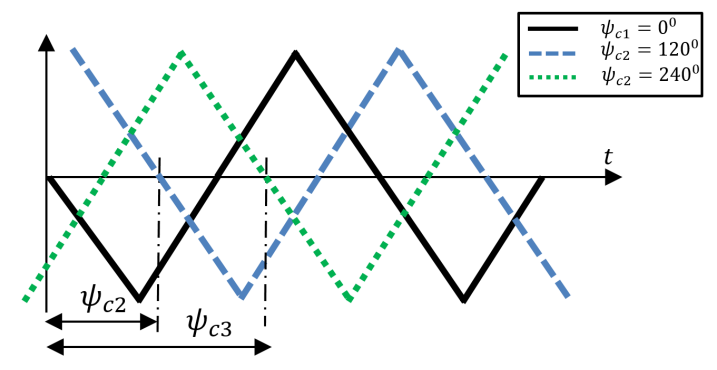

Fig. 2. Shift in the power converters triangular carrier signals

the contribution of each module $(\mu)$ to the voltage across the capacitor can be derived as:

$$
\begin{aligned}
& v_{c \mu}(t)=\frac{3}{2 V_{d} C}\left[\frac{V_{1} I_{m f-2}}{\left(\left(m_{f}-3\right) \omega_{\mathrm{o}}\right)} \cos \left(\left(m_{f}-3\right) \omega_{\mathrm{o}} t+\psi_{c_{\mu}}-\theta_{m f-2}\right)\right. \\
& +\frac{V_{m f-2} I_{1}}{\left(\left(m_{f}-3\right) \omega_{\mathrm{o}}\right)} \cos \left(\left(m_{f}-3\right) \omega_{\mathrm{o}} t+\psi_{c_{\mu}}+\phi\right) \\
& +\frac{V_{m f+2} I_{2 m f-1}}{\left(\left(m_{f}-3\right) \omega_{\mathrm{o}}\right)} \cos \left(\left(m_{f}-3\right) \omega_{\mathrm{o}} t+\psi_{c_{\mu}}-\theta_{2 m f-1}\right) \\
& +\frac{V_{2 m f-1} I_{m f+2}}{\left(\left(m_{f}-3\right) \omega_{\mathrm{o}}\right)} \cos \left(\left(m_{f}-3\right) \omega_{\mathrm{o}} t+\psi_{c_{\mu}}+\theta_{m f+2}\right) \\
& +\frac{V_{1} I_{m f+2}}{\left(\left(m_{f}+3\right) \omega_{\mathrm{o}}\right)} \cos \left(\left(m_{f}+3\right) \omega_{\mathrm{o}} t+\psi_{c_{\mu}}-\theta_{m f+2}\right) \\
& +\frac{V_{m f-2} I_{2 m f+1}}{\left(\left(m_{f}+3\right) \omega_{\mathrm{o}}\right)} \cos \left(\left(m_{f}+3\right) \omega_{\mathrm{o}} t+\psi_{c_{\mu}}-\theta_{2 m f+1}\right) \\
& +\frac{V_{m f+2} I_{1}}{\left(\left(m_{f}+3\right) \omega_{\mathrm{o}}\right)} \cos \left(\left(m_{f}+3\right) \omega_{\mathrm{o}} t+\psi_{c_{\mu}}-\phi\right)+ \\
& +\frac{V_{2 m f+1} I_{m f-2}}{\left(\left(m_{f}+3\right) \omega_{\mathrm{o}}\right)} \cos \left(\left(m_{f}+3\right) \omega_{\mathrm{o}} t+\psi_{c_{\mu}}+\theta_{m f-2}\right) \\
& +\frac{V_{1} I_{2 m f-1}}{\left(\left(2 m_{f}\right) \omega_{\mathrm{o}}\right)} \cos \left(\left(2 m_{f}\right) \omega_{o} t+2 \psi_{c_{\mu}}-\theta_{2 m f-1}\right) \\
& +\frac{V_{1} I_{2 m f+1}}{\left(\left(2 m_{f}\right) \omega_{\mathrm{o}}\right)} \cos \left(\left(2 m_{f}\right) \omega_{\mathrm{o}} t+2 \psi_{c_{\mu}}-\theta_{2 m f+1}\right) \\
& +\frac{V_{m f-2} I_{m f+2}}{\left(\left(2 m_{f}\right) \omega_{\mathrm{o}}\right)} \cos \left(\left(2 m_{f}\right) \omega_{\mathrm{o}} t+2 \psi_{c_{\mu}}-\theta_{m f+2}\right) \\
& +\frac{V_{m f+2} I_{m f-2}}{\left(\left(2 m_{f}\right) \omega_{\mathrm{o}}\right)} \cos \left(\left(2 m_{f}\right) \omega_{\mathrm{o}} t+2 \psi_{c_{\mu}}-\theta_{m f-2}\right) \\
& +\frac{V_{2 m f-1} I_{1}}{\left(\left(2 m_{f}\right) \omega_{\mathrm{o}}\right)} \cos \left(\left(2 m_{f}\right) \omega_{\mathrm{o}} t+2 \psi_{c_{\mu}}-\phi\right) \\
& +\frac{V_{2 m f+1} I_{1}}{\left(\left(2 m_{f}\right) \omega_{\mathrm{o}}\right)} \cos \left(\left(2 m_{f}\right) \omega_{\mathrm{o}} t+2 \psi_{c_{\mu}}+\phi\right) \\
& +\frac{V_{m f-2} I_{2 m f-1}}{\left(\left(3 m_{f}-3\right) \omega_{\mathrm{o}}\right)} \cos \left(\left(3 m_{f}-3\right) \omega_{\mathrm{o}} t+3 \psi_{c_{\mu}}-\theta_{2 m f-1}\right) \\
& +\frac{V_{2 m f-1} I_{m f-2}}{\left(\left(3 m_{f}-3\right) \omega_{\mathrm{o}}\right)} \cos \left(\left(3 m_{f}-3\right) \omega_{\mathrm{o}} t+3 \psi_{c_{\mu}}-\theta_{m f-2}\right) \\
& +\frac{V_{m f+2} I_{2 m f+1}}{\left(\left(3 m_{f}+3\right) \omega_{\mathrm{o}}\right)} \cos \left(\left(3 m_{f}+3\right) \omega_{\mathrm{o}} t+3 \psi_{c_{\mu}}-\theta_{2 m f+1}\right) \\
& +\frac{V_{2 m f+1} I_{m f+2}}{\left(\left(3 m_{f}+3\right) \omega_{\mathrm{o}}\right)} \cos \left(\left(3 m_{f}+3\right) \omega_{\mathrm{o}} t+3 \psi_{c_{\mu}}-\theta_{m f+2}\right) \\
& +\frac{V_{2 m f-1} I_{2 m f+1}}{\left(\left(4 m_{f}\right) \omega_{\mathrm{o}}\right)} \cos \left(\left(4 m_{f}\right) \omega_{\mathrm{o}} t+4 \psi_{c_{\mu}}-\theta_{2 m f+1}\right) \\
& \left.+\frac{V_{2 m f+1} I_{2 m f-1}}{\left(\left(4 m_{f}\right) \omega_{\mathrm{o}}\right)} \cos \left(\left(4 m_{f}\right) \omega_{\mathrm{o}} t+4 \psi_{c_{\mu}}-\theta_{2 m f-1}\right)\right]
\end{aligned}
$$

In (6) $m$ is the carrier index and $n$ is the base band index
TABLE II

HARMONIC CONTENT IN THE DC BUS VOLTAGE RIPPLE AND, FOR EACH HARMONIC, PHASE COMPONENT DUE TO THE SHIFT $\psi_{c_{\mu}}$ OF THE CARRIER SIGNAL

\begin{tabular}{|c|c|}
\hline $\mathbf{h}$ & Phase component due to shift $\psi$ \\
\hline$m_{f} \pm 3$ & $\psi_{c_{\mu}}$ \\
\hline $2 m_{f}$ & $2 \psi_{c_{\mu}}$ \\
\hline $3 m_{f} \pm 3$ & $3 \psi_{c_{\mu}}$ \\
\hline $4 m_{f}$ & $4 \psi_{c_{\mu}}$ \\
\hline
\end{tabular}

for the $h$ harmonic component, due to the SPWM modulation: $h=m \cdot m_{f}+n$, the product $V_{h n} I_{h m}$ is between two harmonic amplitudes of voltage and current, $\phi$ the displacement between voltage and current fundamental components, $\delta$ the load angle, $m_{f}$ the SPWM frequency modulation ratio, $\omega_{o}$ the output fundamental angular frequency, $\psi_{c_{\mu}}$ the phase shift applied to the carrier signal. $V_{h n}$ is the voltage harmonic amplitudes. $I_{h m}$ and $\theta_{h m}$ are the current harmonic amplitudes and their characteristic angles, respectively. They are obtained by applying each voltage harmonic $V_{h m}$, calculated in [7], to the equivalent $R-L-e$ circuit of the AFPMSG.

The analysis is limited to the same number of harmonics considered in [7]. Also each group of terms of the same harmonic order is function of distinct multiple of the shift in the converters triangular carrier signal $\psi_{c_{\mu}}$. In fact the same guidelines provided in [7] are applied here. Table II summarizes the harmonics present in the voltage expression of (6). It is evident from (6) that the expression of the dc bus voltage harmonic reflecting a classical SPWM can be evaluated by setting $\psi_{c_{\mu}}=0$ for all three converters.

\section{SPWM AND SEQUential Command}

In order to eliminate the least order harmonic, $m_{f}-3$, with three modules paralleled, the shift angles $\psi_{c_{n}}$ have to be $0^{\circ}$, $120^{\circ}, 240^{\circ}$ respectively as shown in Fig. 2. Such a selection of the angles will also eliminate the harmonics of order $m_{f}+3$, $2 m_{f}$ and $4 m_{f}$ [7].

The value of $m_{f}$ should be kept low in order to limit switching losses. However, for the sake of the current study, higher values of $m_{f}$ are considered too. The total harmonic distortion is calculated using (6) at different values of $m_{f}$ for the whole speed range.

Fig. 3 represents the Total Harmonic Distortion in the DC bus voltage $\left(T H D_{V}\right)$ over the whole speed range of the operation, i.e. from the cut-in frequency $\left(f_{\text {cutin }}\right)$ to the rated one $\left(f_{\text {rated }}\right)$. It is evident that a great reduction in the $T H D_{V}$ can be achieved using sequential command. For example, in case $m_{f}=15$ with sequential command has a lower $T H D_{V}$ than $m_{f}=69$ without sequential command. Additionally, considering near-rated conditions, $m_{f}=15$ also has a lower TH $D_{V}$ compared to $m_{f}=99$ and $m_{f}=135$.

Figs. 4, 5 shows the behaviour of $K_{p}$ over the whole frequency range. Both the increase of $m_{f}$ and the application of sequential command decrease $K_{p}$.

Figs. 6, 7 show the behaviour of $K_{s}$ over the whole frequency range. In this case, increasing $m_{f}$ does not affect 


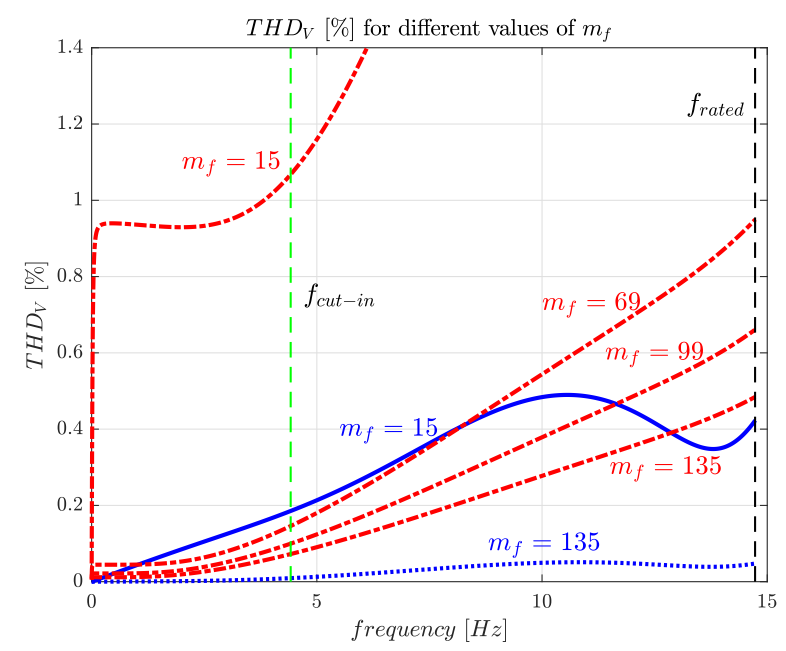

Fig. 3. DC bus voltage $T H D_{V} \%$ with three-module machine with sequential command $\left(m_{f}=15,135\right.$ in blue) and without sequential command $\left(m_{f}=\right.$ $15,69,99,135$ in red). Results are obtained from (6)

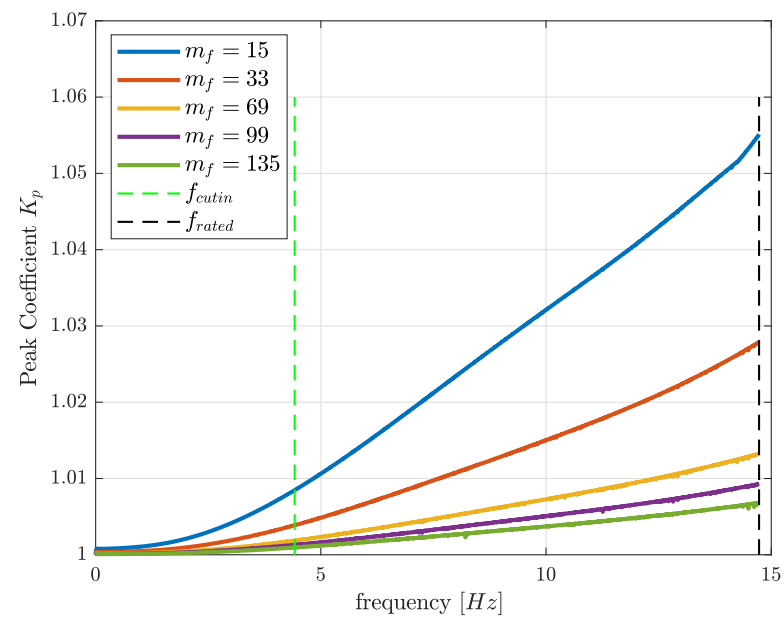

Fig. 4. Peak coefficient $K_{p}$ for different values of $m_{f}$ for classical SPWM. $K_{p}$ is proportional to the machine frequency and inversely proportional to $m_{f}$. In order to limit $K_{p}$ when operating at higher frequencies, a higher $m_{f}$ is necessary, though, at the cost of increased switching losses.

$K_{s}$ so much. Increasing $m_{f}$ decreases the voltage harmonics amplitudes, as reflected in the behaviour of $K_{p}$. As regards $K_{s}$, the harmonic content is only shifted at higher order harmonics; thus only with sequential command a reduction in $K_{s}$ is possible by eliminating some other harmonics. This is particularly evident for higher values of $m_{f}$.

\section{Simulation Results And Discussion}

The system presented in Section III-A is implemented in Matlab/Simulink. Each module is modelled by standard two axis d-q model of PMSM. For each module the classical FOC is implemented, the machine is vector-controlled to operate in maximum torque-per-ampere (MTPA) conditions. The DC bus voltage is kept at $V_{d n}$.

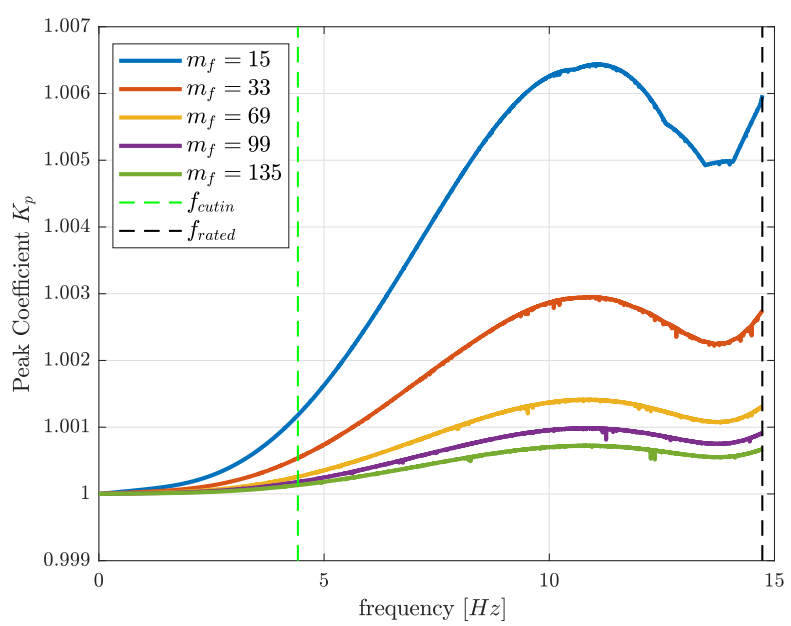

Fig. 5. Peak coefficient $K_{p}$ for different values of $m_{f}$ with Sequential Command. As opposite to classical SPWM, it is possible to operate at higher frequency with lower $m_{f}$ and comparable values of $K_{p}$.

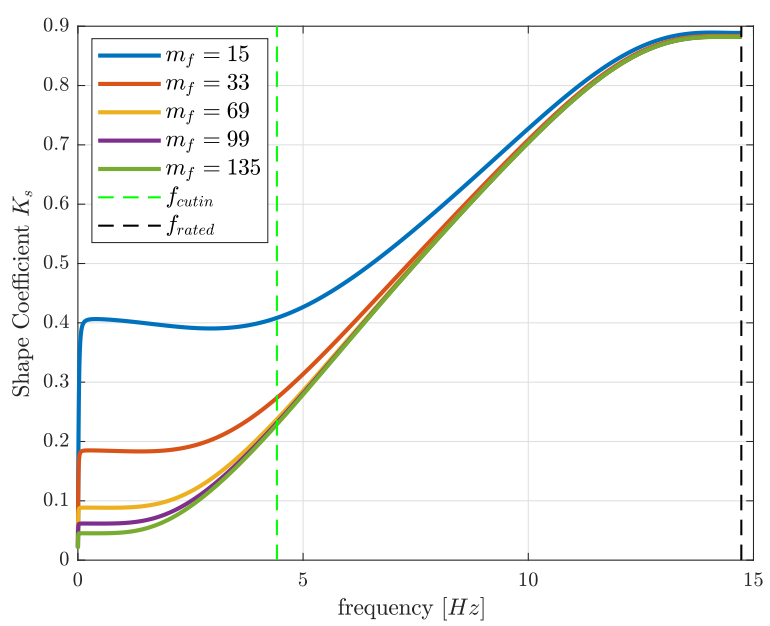

Fig. 6. Shape coefficient $K_{s}$ for different values of $m_{f}$ for classical SPWM. At lower frequencies it is possible to limit $K_{s}$ simply by increasing $m_{f}$. However as frequency increases, the benefits of a higher $m_{f}$ become less and less evident.

For the wind plant simulation, real data on the wind speeds are considered. Table III reports the 2008 monthly average wind speeds collected in the Kentish Flats Offshore Wind Farm [12], the relative machine frequencies, i.e., each wind speed has an associated frequency induced on the DC side by the machine torque oscillations. The relevant THD obtained from Matlab/Simulink simulations, with and without sequential command, are also reported for the entire year. The analysis is limited to two values of $m_{f}, m_{f}=15$ and $m_{f}=69$.

\section{A. Theoretical Model Validation}

Several simulations are carried out for the wind speeds reported in Table III in order to validate the accuracy of the 


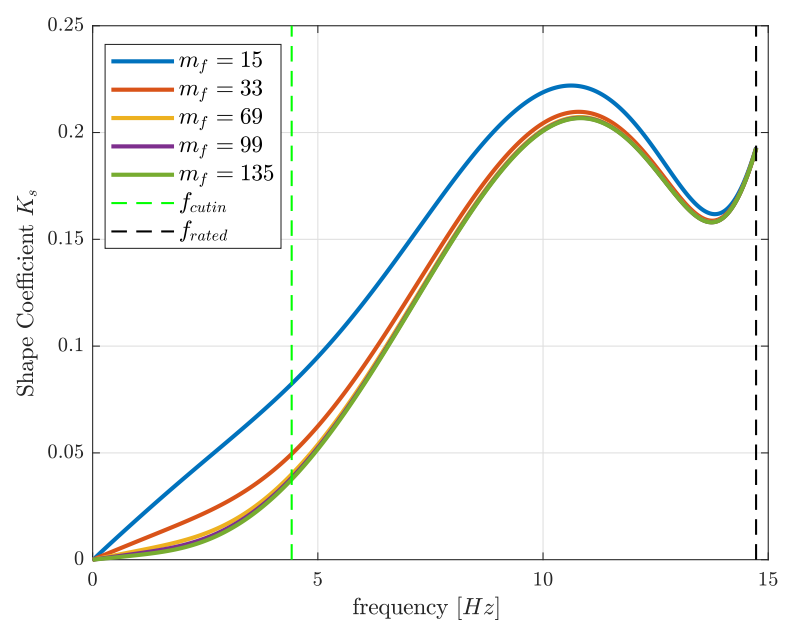

Fig. 7. Shape coefficient $K_{s}$ for different values of $m_{f}$ with Sequential Command. Sequential Command is able to reduce $K_{s}$ over the whole frequency range.

TABLE III

YEARLY THD $\%$ PROFILES IN KENTISH FLATS WITH $(w /)$ AND WITHOUT $(w / o)$ SEQUENTIAL COMMAND FOR $m_{f}=15,69$, UNDER DIFFERENT AVERAGE WIND SPEEDS $\left(v_{w i n d}\right)$ AND MACHINE FREQUENCIES $\left(f_{e}\right)$

\begin{tabular}{|c|c|c|c|c|c|c|}
\hline Month & $\begin{array}{c}\mathbf{v}_{\mathbf{w i n d}} \\
{[\mathbf{m} / \mathbf{s}]}\end{array}$ & $\begin{array}{c}\mathbf{f}_{\mathbf{e}} \\
{[\mathbf{H z}]}\end{array}$ & $\begin{array}{c}\mathbf{T H D}_{\mathbf{w} / \mathbf{o}} \\
{[\%]} \\
m_{f}=15\end{array}$ & $\begin{array}{c}\mathbf{T H D}_{\mathbf{w} /} \\
{[\%]}\end{array}$ & $\begin{array}{c}\mathbf{T H D}_{\mathbf{w} / \mathbf{o}} \\
m_{f}=15\end{array}$ & $\begin{array}{c}\mathbf{T H D}_{\mathbf{w} /} \\
m_{f}=69\end{array}$ \\
$\begin{array}{c}{[\%]} \\
m_{f}=69\end{array}$ \\
\hline JAN & 11 & 11.9 & 3.49 & 0.50 & 0.73 & 0.11 \\
\hline FEB & 8 & 8.7 & 2.37 & 0.51 & 0.50 & 0.10 \\
\hline MAR & 10.1 & 10.9 & 3.15 & 0.56 & 0.66 & 0.11 \\
\hline APR & 7.5 & 8.1 & 2.15 & 0.47 & 0.45 & 0.09 \\
\hline MAY & 7.5 & 8.1 & 2.15 & 0.47 & 0.45 & 0.09 \\
\hline JUN & 6.2 & 6.7 & 1.65 & 0.37 & 0.34 & 0.06 \\
\hline JUL & 6.9 & 7.5 & 1.94 & 0.43 & 0.40 & 0.08 \\
\hline AUG & 8 & 8.7 & 2.37 & 0.51 & 0.50 & 0.10 \\
\hline SEP & 7.3 & 7.9 & 2.08 & 0.46 & 0.44 & 0.09 \\
\hline OCT & 8 & 8.7 & 2.37 & 0.51 & 0.50 & 0.10 \\
\hline NOV & 8.9 & 9.6 & 2.69 & 0.56 & 0.57 & 0.11 \\
\hline DEC & 7.9 & 8.6 & 2.33 & 0.51 & 0.50 & 0.11 \\
\hline
\end{tabular}

mathematical model, (6), all quantities are steady state ones.

Fig. 8 shows the DC bus voltage for a three-module machine with and without the sequential command at $f_{\text {rated }}$. A significant reduction of the harmonics content is evident. Also Table III verifies the effectiveness of the sequential command in decreasing $T H D_{V}$.

Fig. 9 represents the $T H D_{V}$ in the DC bus voltage over the whole speed range of the operation, i.e. from the cutin speed to the rated speed for both values of $m_{f}$. Two remarks can be made; it is evident that a great reduction in the $T H D_{V}$ can be achieved using sequential command. Also, it is important to notice that theoretical results obtained from (6) well approximate the simulated behaviour.

\section{B. Electric stresses coefficients}

Since all the quantities under study are in DC, the machine frequency related to the considered average wind speed is taken as the fundamental frequency in the shape coefficient, $K_{s}$, calculations.

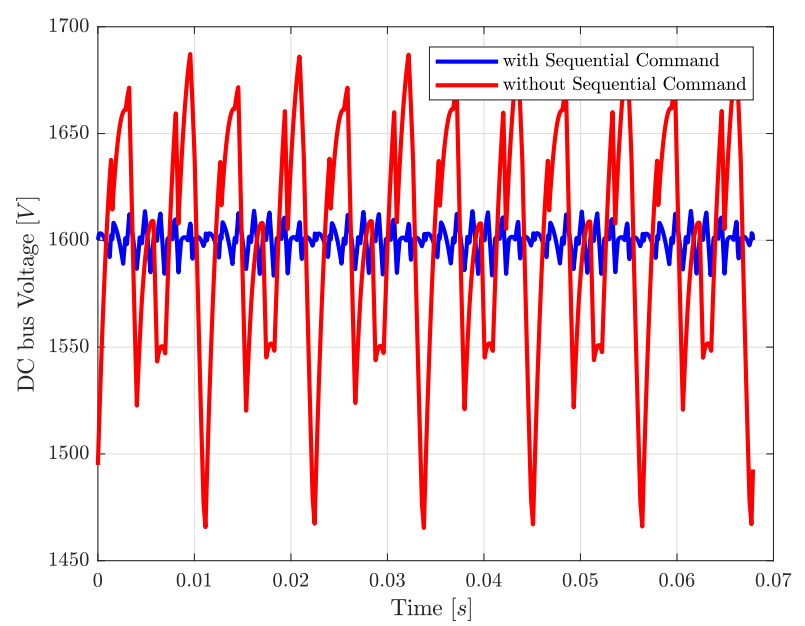

Fig. 8. Steady state DC bus voltage with and without sequential control. The harmonic content in the voltage waveform is much lower than without the sequential control
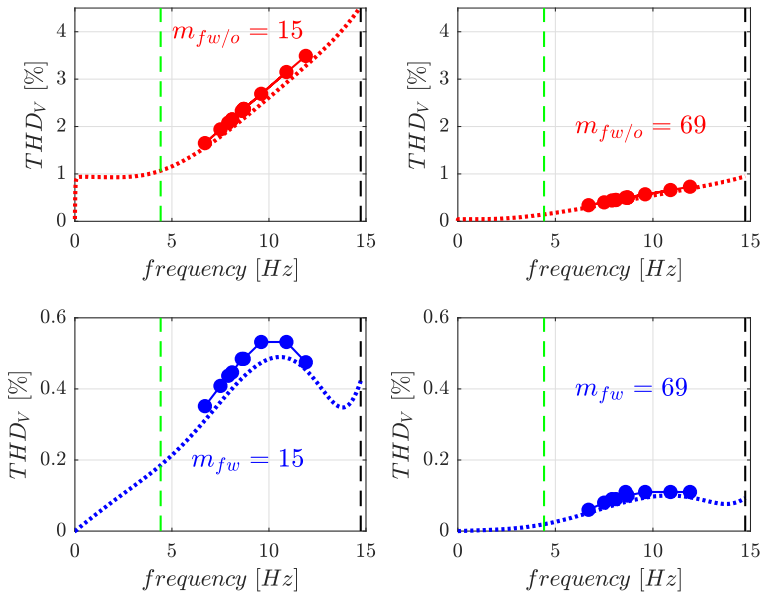

Fig. 9. A comparison between simulated results (solid line) of the DC bus voltage $T H D_{V} \%$ and those obtained through the theoretical approach (dotted line) for $\left(m_{f}=15\right.$ and $m_{f}=69$ ) with (top plots in red) and without (bottom plots in blue) sequential command

Fig. 10 shows $K_{p}$ over the one year period with the two different control strategies. Both increasing $m_{f}$ and applying sequential command have reduced $K_{p}$ in an effective way. Fig. 11 shows $K_{s}$ : in this case, only by changing the modulation technique $K_{s}$ is reduced.

Finally, since electric stress is a cumulative phenomenon over time, the significant reduction in the coefficients obtained with sequential command means, according to (4), an increase in the lifetime of the capacitor.

Fig. 12 compares the values obtained both with a Fourier Transform (FT) analysis, i.e., as in (3) , applied to the simulation results, and with a theoretical approach, i.e., by means of 6 .

The resulting difference is due to two main causes: 

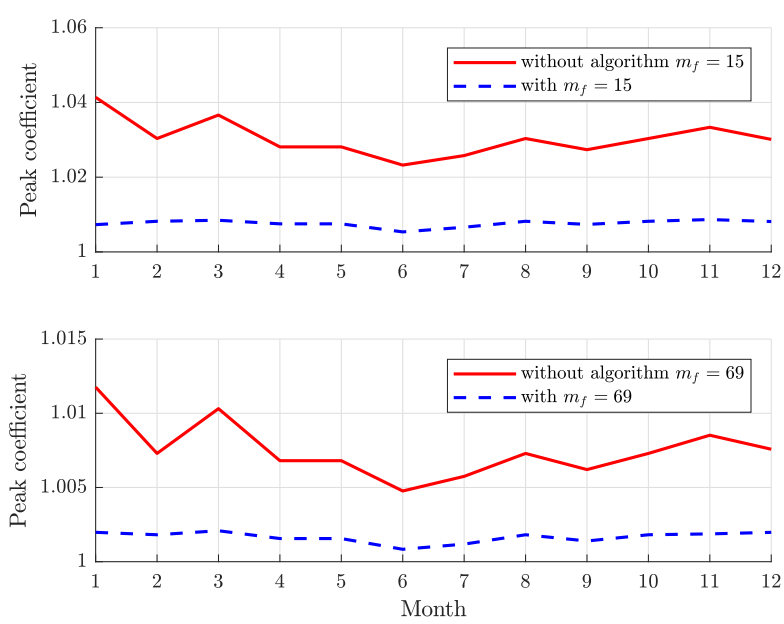

Fig. 10. Yearly profile of the peak coefficient $K_{p}$ for $m_{f}=15$ (top graph) and $m_{f}=69$ (bottom graph) with (dotted in blue) and without (solid in red) sequential command. Both increasing $m_{f}$ or using Sequential Command instead of SPWM reduce $K_{p}$
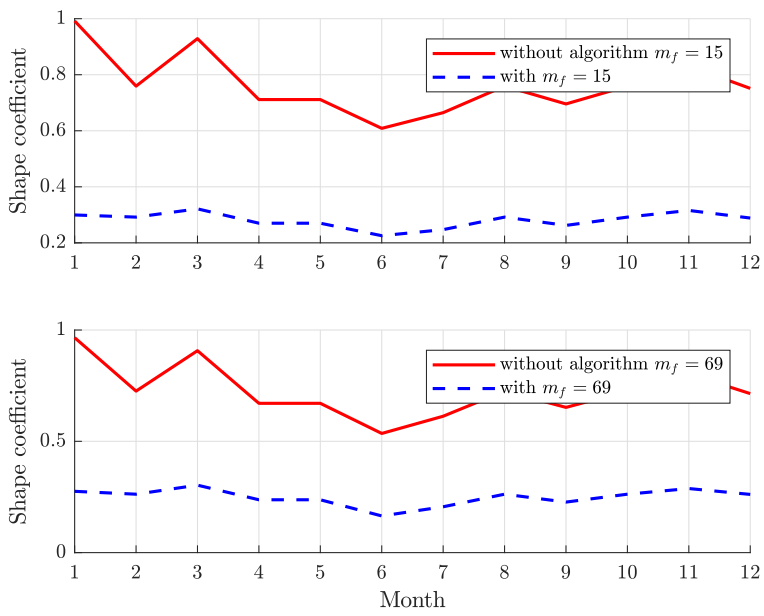

Fig. 11. Yearly profile of the shape coefficient $K_{s}$ for $m_{f}=15$ (top graph) and $m_{f}=69$ (bottom graph) with (dotted in blue) and without (solid in red) sequential command. Only using Sequential Command instead of SPWM reduces $K_{p}$ : in fact, increasing $m_{f}$ has no effect on $K_{s}$.

- the theoretical approach proposed in (6) considers a limited number of harmonics while the FT considers the whole spectrum.

- leakage errors, because of non-synchronous sampling conditions, affect the resulting estimates [13].

\section{CONCLUSIONS}

This paper analyses the electric stress on the DC bus capacitor in a three-module AFPMSG wind energy system. The modular configuration allows (i) the usage of off-theshelf converters, (ii) the shift of the carrier signals among the parallel converters with a significant reduction in the ripple components of the DC voltage. The simulation results under-
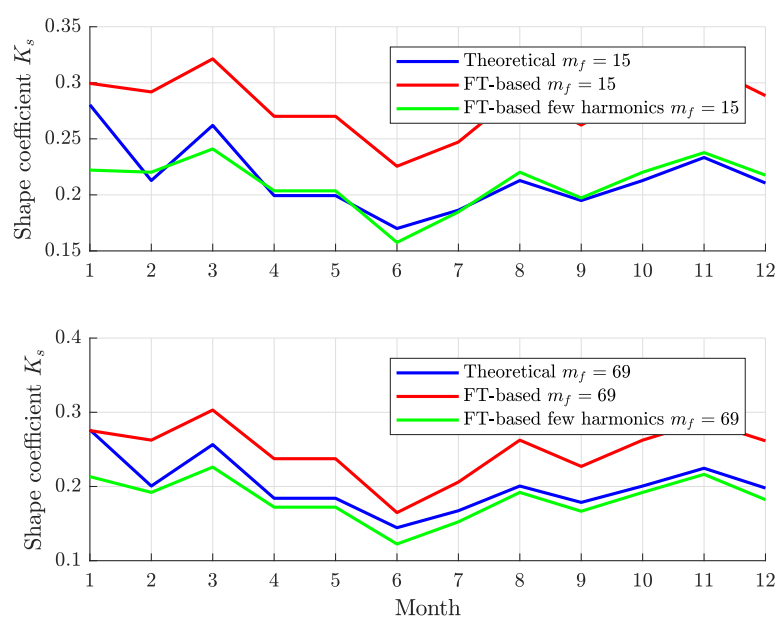

Fig. 12. Comparison between the electric stress shape coefficient $K_{s}$ with $m_{f}=15$ (top graph) and $m_{f}=69$ (bottom graph), as obtained from the theoretical approach (in blue), the FT analysis considering the whole spectrum (in red) and the FT analysis considering the same harmonics as in the theoretical approach (in green)

line how classical strategies may prove sub-optimal because of the higher stress induced and, thus, result in a reduction of dielectric's residual useful life. Future work will expand this first investigation on power converters control schemes using as a benchmark the electric stress on dielectrics.

\section{REFERENCES}

[1] "IEEE recommended practice for monitoring electric power quality," IEEE Std 1159-2009 (Rev. IEEE Std 1159-1995), pp. c1-81, June 2009.

[2] A. Cavallini, D. Fabiani, and G. C. Montanari, "Power electronics and electrical insulation systems - part 1: Phenomenology overview," IEEE Elect. Ins. Mag., vol. 26, no. 3, pp. 7-15, May 2010.

[3] — , "Power electronics and electrical insulation systems - part 2: life modeling for insulation design," IEEE Elect. Ins. Mag., vol. 26, no. 4, pp. 33-39, July 2010.

[4] — "Power electronics and electrical insulation systems - part 3: Diagnostic properties," IEEE Elect. Ins. Mag., vol. 26, no. 5, pp. 30-40, September 2010.

[5] K. Lee, T. M. Jahns, T. A. Lipo, G. Venkataramanan, and W. E. Berkopec, "Impact of input voltage sag and unbalance on dc-link inductor and capacitor stress in adjustable-speed drives," IEEE Trans. on Ind. Appl., vol. 44, no. 6, pp. 1825-1833, Nov 2008.

[6] Y. Yang, K. Ma, H. Wang, and F. Blaabjerg, "Mission profile translation to capacitor stresses in grid-connected photovoltaic systems," in 2014 IEEE Conf. ECCE, Sept 2014, pp. 5479-5486.

[7] A. Di Gerlando, K. El Shawarby, G. M. Foglia, M. F. Iacchetti, and R. Perini, "DC current and torque ripple mitigation in modular PMSG drives for multi-MW WECSs with linear PWM inverter modulation," in submitted to 2018 IEEE Conf. ICEM.

[8] A. S. Farag, C. Wang, T. C. Cheng, G. Zheng, Y. Du, L. Hu, B. Palk, and M. Moon, "Failure analysis of composite dielectric of power capacitors in distribution systems," IEEE Trans. on Diel. and Elect. Ins., vol. 5, no. 4, pp. 583-588, Aug 1998.

[9] D. A. Hewitt, J. E. Green, J. N. Davidson, M. P. Foster, and D. A. Stone, "Observation of electrolytic capacitor ageing behaviour for the purpose of prognostics," in 2016 IEEE Conf. IECON, Oct 2016, pp. 2195-2200.

[10] A. Carboni, G. Perrone, E. Ragaini, and A. Ferrero, "The path towards smarter circuit breakers," in submitted to 2018 IEEE Conf. AMPS.

[11] “ABB Ability," http://new.abb.com/abb-ability.

[12] http://wind-power-program.com.

[13] G. D'Antona and A. Ferrero, Digital Signal Processing for Measurement Systems. Theory and Applications. New York, NY, USA: Springer, 2006. 